\title{
High-brightness Phosphor-conversion White Light Source Using InGaN Blue Laser Diode
}

\author{
Han-Youl Ryu* and Dae-Hwan Kim \\ Department of Physics, Inha University, Incheon 402-751, Korea
}

(Received July 12, 2010 : revised October 11, 2010 : accepted October 12, 2010)

\begin{abstract}
A phosphor-conversion white light source is demonstrated using an InGaN-based blue laser diode (LD) and a yellow-emitting phosphor excited by the blue LD. The photometric and colorimetric properties of this blue-LD-based white light source are characterized. When injection current of the LD is $100 \mathrm{~mA}$, luminous flux and luminous efficiency of the white light are found to be over $5 \mathrm{~lm}$ and $10 \mathrm{~lm} / \mathrm{W}$, respectively. When injection current is $>90 \mathrm{~mA}$, luminance is estimated to be larger than $10 \mathrm{Mcd} / \mathrm{cm}^{2}$. In addition, color characteristics of the white light such as chromaticity coordinates, a correlated color temperature, and a color rendering index are found to be quite stable as current and temperature of the LD varies. The demonstrated LD-based white light source is expected to be used in high-brightness illumination applications with good color stability.
\end{abstract}

Keywords: White light, Blue laser diode, Light-emitting diode, High brightness, Colorimetry

OCIS codes : (140.5960) Semiconductor lasers; (260.3800) Luminescence; (330.1710) Color measurement

\section{INTRODUCTION}

GaN-based light-emitting diodes (LEDs) have found various applications such as traffic signals, automobile lamps, back lights in liquid-crystal displays, and illumination systems $[1,2]$. In particular, solid-state lighting based on the $\mathrm{GaN}$ LEDs has attracted great interest as a high-energy-efficiency and environment-friendly lighting technology. The applications of GaN-based white LEDs toward general lighting require high-power and high-efficiency operation. Recently developed LEDs have demonstrated high-power of $>1 \mathrm{~W}$ and high-efficiency of $>100 \mathrm{~lm} / \mathrm{W}[3,4]$. In some illumination applications, small-size and high-brightness light sources are required. Due to the small source size, LEDs are well suited for such applications [3-5]. In photometry, source brightness corresponds to the luminance of devices which is defined as the ratio of luminous flux to the source étendue [2]. Therefore, it is important to manufacture white light sources with small light-emitting area and low beam divergence as well as high output power for realizing high brightness sources.

One interesting example of the high-brightness white light sources is a phosphor-conversion white light based on an InGaN laser diode (LD) and phosphors excited by the
LD, which was first demonstrated by Nichia [6]. Due to the small emission spot size, this LD-based white light source was named 'Micro White' and high luminance is expected at relatively low operation power. Two types of LD-based white light sources have been introduced. One is based on a 405-nm violet LD and blue/yellow phosphors $[6,7]$, and another is using a 450-nm blue LD and a yellow phosphor [8]. The mechanism of white light emission in these LD-based white sources is basically similar to that of phosphor-conversion white LEDs. In the violet-LD-based white light, a 405-nm LD excites both blue and yellow phosphors to emit blue and yellow light that is mixed to produce white light. In the blue-LD-based white light, part of the 450-nm blue laser light is absorbed in the yellow phosphor and the unabsorbed blue laser light is transmitted outside the phosphor. The yellow light emitted from the phosphor is mixed with transmitted blue laser light to appear as white light.

In principle, the blue-LD-based white light shows higher efficiency than the violet-LD-based one because the Stoke's shift loss during wavelength conversion in the phosphor is lower. Stoke's shift loss at the phosphor increases as the wavelength difference between excitation light of a pump source and emission light from the phosphor increases. In

\footnotetext{
*Corresponding author: hanryu@inha.ac.kr

Color versions of one or more of the figures in this paper are available online.
} 
addition, the transmitted blue laser light can be used for high-speed data communication by modulating the LD. Although the blue LD-based white light source was first introduced by Nichia several years ago [8], the characteristics of this white light has not been presented in detail compared to those of violet-LD-based white light $[6,7]$. In this paper, we experimentally demonstrate the blue-LD based white light, and investigate its photometric and colorimetric properties such as luminous flux, chromaticity coordinates, correlated color temperature (CCT), and a color rendering index (CRI).

\section{EXPERIMENT}

An LD-based phosphor-conversion white light source was assembled using an InGaN blue LD and an Eu-doped silicate $\left[(\mathrm{BaSr})_{2} \mathrm{SiO}_{4}: \mathrm{Eu}^{2+}\right]$ yellow phosphor. The structure and characteristics of the blue LD are similar to those reported in Refs. [9-11]. The multiple-quantum-well (MQW) active layers of the LD are composed of two pairs of a 25 $\AA$-thick $\operatorname{In}_{0.16} \mathrm{Ga}_{0.84} \mathrm{~N}$ wells that were designed to emit blue light around $445 \mathrm{~nm}$. The configuration of the white light system is basically similar to that shown in Ref. [8]. The blue laser light was coupled into one end of a plastic optical fiber with core diameter of $400 \mu \mathrm{m}$. The other end of the optical fiber was covered with some amount of the yellow phosphor. When the phosphor was excited by the blue LD, yellow light generated at the phosphor was combined with blue laser light to emit white light.

The output characteristics of the LD were measured under continuous-wave operation with varying temperatures of the LD. The operation temperature of the LD was adjusted by a thermo-electrically controlled module. Peak emission wavelength exists around $445 \mathrm{~nm}$ above threshold at $20^{\circ} \mathrm{C}$. Fig. 1(a) shows output power versus current $(L-I)$ curves of the LD for several temperatures from 20 to 8 $0^{\circ} \mathrm{C}$. At $20^{\circ} \mathrm{C}$, threshold current and slope efficiency are $\sim 35 \mathrm{~mA}$ and $\sim 0.7 \mathrm{~W} / \mathrm{A}$, respectively. Good linearity of the $L-I$ curve from threshold to output power of $>50 \mathrm{~mW}$ is observed, and the $L-I$ curve does not exhibit roll-over up to $120 \mathrm{~mA}$ even at high temperature of $80^{\circ} \mathrm{C}$. Fig. 1(b) shows voltage and wall plug efficiency (WPE) of the LD as a function of current at $20^{\circ} \mathrm{C}$. Due to the nonlinear characteristic of the voltage-current relation, the WPE increases sublinearly with current. The WPE does not decrease in the measurement range. Operation current and voltage at $50-\mathrm{mW}$ output power are $\sim 105 \mathrm{~mA}$ and $\sim 5.43$ $\mathrm{V}$, respectively, resulting in the WPE of $8.7 \%$.

The characteristics of the assembled white light were measured by using a spectro-radiometric system by Ocean Optics, Inc [12]. The white light emitted from the optical fiber was collected by an integrating sphere and coupled into a spectrometer for photometric and colorimetric measurements. Spectral data were analyzed based on the standard by Commission International de I'Eclairage (CIE) to evaluate
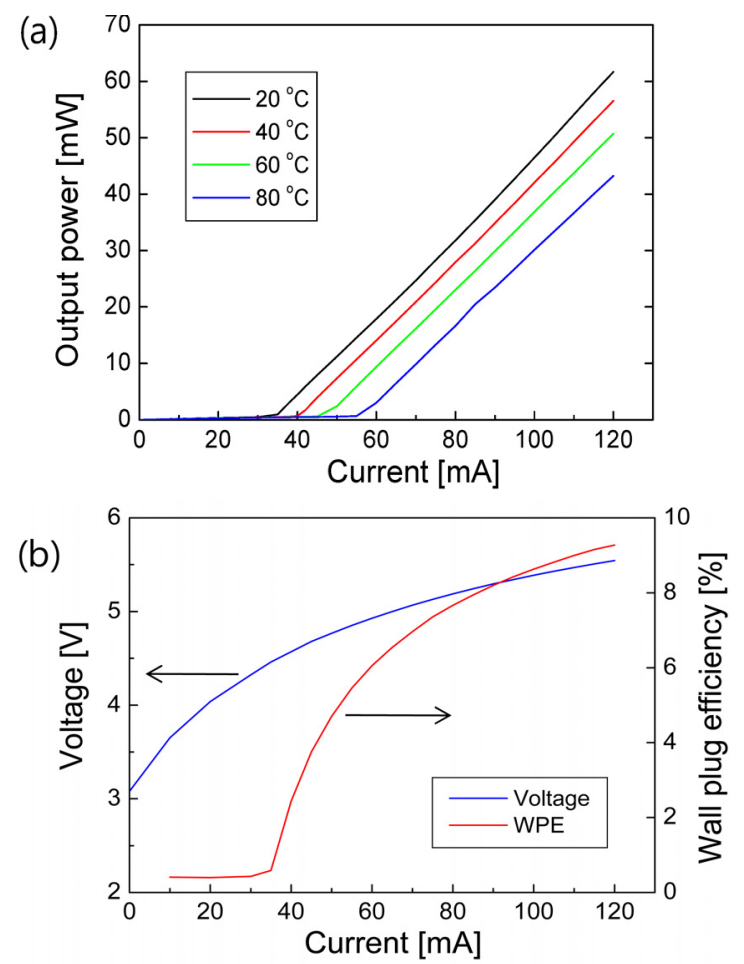

FIG. 1. (a) Light-current ( $L-I)$ curves of the blue laser diode (LD) for temperatures from 20 to $80^{\circ} \mathrm{C}$. (b) Voltage and wall plug efficiency (WPE) of the LD as a function of current when temperature is $20^{\circ} \mathrm{C}$.

luminous flux, illuminance, chromaticity coordinates, a CCT, and a CRI. We measured the color data with changing operation current and temperature of the LD to investigate the stability of the white light.

\section{RESULTS AND DISCUSSION}

Fig. 2 shows a spectrum of the white light source measured at $20^{\circ} \mathrm{C}$ when injection current is $40 \mathrm{~mA}$. The sharp peak around $445 \mathrm{~nm}$ corresponds to laser light transmitted through the phosphor, and a broad spectral region from $500 \mathrm{~nm}$ to $700 \mathrm{~nm}$ originates from the phosphor excited by the LD. The peak wavelength and full-width at halfmaximum of the spectrum by the phosphor is $563 \mathrm{~nm}$ and $87 \mathrm{~nm}$, respectively, corresponding to yellow-green color. Proper combination of the blue and the yellow light gives white light as in usual white LEDs [2]. Since spectral linewidth of the blue light is much narrower than that of the yellow light, peak intensity of the blue laser light should be higher by more than 3 times to exhibit white light.

A typical $L-I$ curve of the LD-based white light is shown in Fig. 3(a). Here, operation temperature of the LD was 2 $0^{\circ} \mathrm{C}$. The features of the $L-I$ curve basically reflect the $L-I$ curve of the LD shown in Fig. 1. Threshold current of 35 $\mathrm{mA}$ is the same as the threshold of the LD and the $L-I$ 


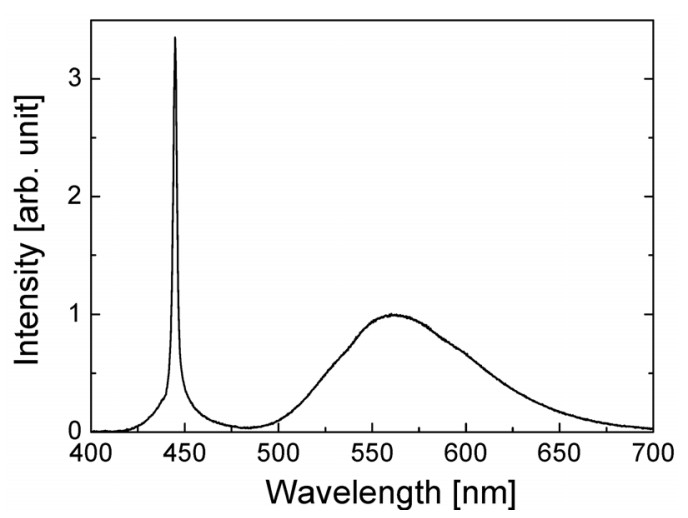

FIG. 2. Emission spectrum of the white light from a blue LD and a yellow phosphor measured at $20^{\circ} \mathrm{C}$ when injection current of the LD is $40 \mathrm{~mA}$.

(a)

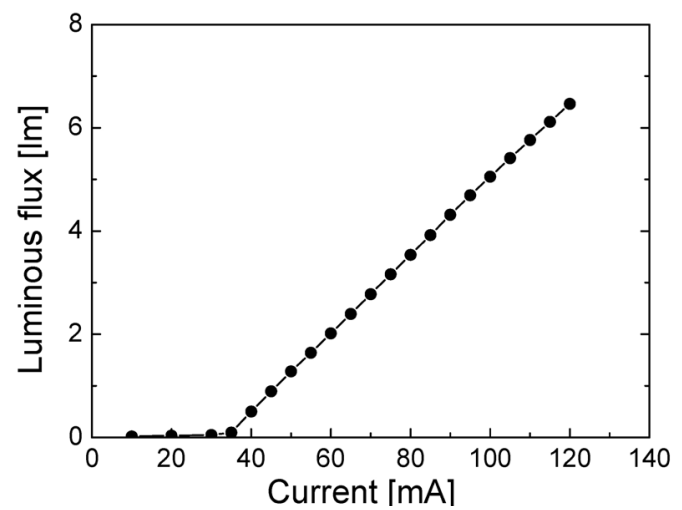

(b)

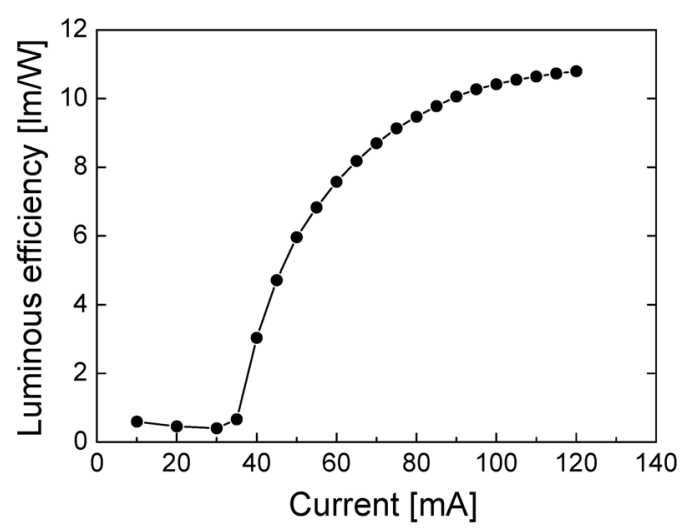

FIG. 3. $L-I$ curve of the white light source when operation temperature of the $\mathrm{LD}$ is $20^{\circ} \mathrm{C}$. Good linearity of the $L-I$ curve is observed.

curve of the white light shows good linearity as in the $L-I$ curve of the LD. In this white light, slope efficiency is 75 $\mathrm{lm} / \mathrm{A}$ and luminous flux at $100 \mathrm{~mA}$ is about $5 \mathrm{~lm}$. The good linearity of the $L-I$ curve implies that the response of the yellow phosphor is almost constant and does not change with input LD power.

Luminous efficiency of the white light is shown in Fig. 3(b) as a function of injection current of the LD. It increases sublinearly with current and becomes $>10 \mathrm{~lm} / \mathrm{W}$ when the injection current is $>90 \mathrm{~mW}$. The efficiency curve of the white light is basically similar to that of the WPE of the LD shown in Fig. 1(b). Note that the luminous efficiency does not decrease even at high injection current of $>100$ $\mathrm{mA}$. This is in strong contrast to the case of usual white LEDs where efficiency begins to decrease substantially as injection current increases. This 'efficiency droop' phenomenon has limited high-power and high-efficiency operation of LEDs, and there have been controversial issues on the mechanisms and remedies for the efficiency droop [4, 13-16]. On the contrary, the LD-based white light does not undergo the efficiency droop problem. This basically results from the fact that the LD does not exhibit the efficiency droop due to the good linearity of the $L-I$ curve as shown in Fig. 1.

However, the luminous efficiency of the demonstrated white light source is too low compared to $>100 \mathrm{~lm} / \mathrm{W}$ luminous efficiency of up-to-date high-efficiency white LEDs. There are several reasons for this low efficiency. First, power-conversion efficiency of the LD is quite low. The WPE of the LD at $100 \mathrm{~mA}$ was only $8.5 \%$. Second, there is energy loss during wavelength conversion at phosphor from blue to yellow light. This Stoke's shift loss is estimated to be $\sim 20 \%$. Considering that the maximum theoretical efficiency of white light is $\sim 300 \mathrm{~lm} / \mathrm{W}$, these two effects reduce the luminous efficiency to $\sim 20 \mathrm{~lm} / \mathrm{W}$. Third, the remaining loss was caused during optical coupling from the LD to the fiber and the fiber to the spectro-radiometer. By comparing the measured luminous efficiency and the two loss-causing effects mentioned above, the coupling losses are estimated to be $\sim 50 \%$. Therefore, the luminous efficiency can be increased by improving the performance of the blue LD and the coupling efficiency of white light. Recently reported WPE of high-power blue LDs can be as high as $24 \%$ at 1000-mW output power [17]. By using such high-power and high-performance blue LDs, luminous efficiency of the LD-based white light source will increase substantially.

Luminance of the white light source can be estimated from the measured luminous flux by using the relations between photometric quantities [18]. First, luminous exitance of the white light is determined by dividing the luminous flux by source area. Assuming that the area of the white light source is the same as the area of the fiber, the luminous exitance on the $400-\mu \mathrm{m}$-diameter fiber is obtained to be $\sim 4 \times 10^{7} \mathrm{~lm} / \mathrm{m}^{2}$ when luminous flux is $5 \mathrm{~lm}$. Second, luminance of the white light can be determined from the luminous exitance. For a perfect Lambertian surface, luminous exitance is equal to $\pi$ times luminance. Consequently, luminance is estimated to be $12.7 \mathrm{Mcd} / \mathrm{m}^{2}$ for the luminous exitance of $4 \times 10^{7} \mathrm{~lm} / \mathrm{m}^{2}$. The luminance of our white light source becomes $>10 \mathrm{Mcd} / \mathrm{m}^{2}$ when luminous flux is higher than $4 \mathrm{~lm}$ or injection current is larger than $90 \mathrm{~mA}$. High-brightness white light with luminance of $>10 \mathrm{Mcd} / \mathrm{m}^{2}$ has also been demonstrated 
from several light sources such as high-power LEDs, halogen filament lamps, and high-intensity discharge (HID) arc lamps [2]. However, these high-luminance light sources operate at high luminous flux of $>100 \mathrm{~lm}$, which requires high input electrical power. In our LD-based white light source, in contrast, high luminance can be achieved with relatively low input power. This property can be used in some specific applications that require low-power, small-size, and high luminance light sources.

Next, color characteristics of the white light source are investigated. Fig. 4 and 5 show color data such as chromaticity coordinates, a CCT, and a CRI as a function of injection current and temperature of the LD, respectively. Here, chromaticity coordinates in the CIE $x y$ chromaticity diagram are plotted. In Fig. 4, current was increased from $40 \mathrm{~mA}$ to $80 \mathrm{~mA}$ at fixed temperature of $20^{\circ} \mathrm{C}$. In Fig. 5 , operation temperature of the LD was increased from 20 to $80^{\circ} \mathrm{C}$ when $\mathrm{LD}$ output power was $20 \mathrm{~mW}$. The range of chromaticity coordinates are $0.35 \sim 0.36$ and that of CCT are $4200 \sim 4300 \mathrm{~K}$, which well represent the properties of white light. CRI is foundto be less than 60 , somewhat lower than the CRI of conventional white light sources such as white LEDs and fluorescent lamps. The low CRI results from the narrow spectrum in the blue wavelength

(a)

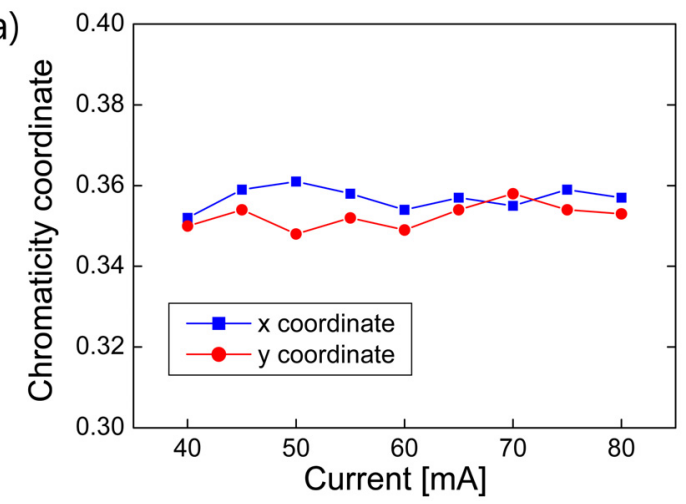

(b)

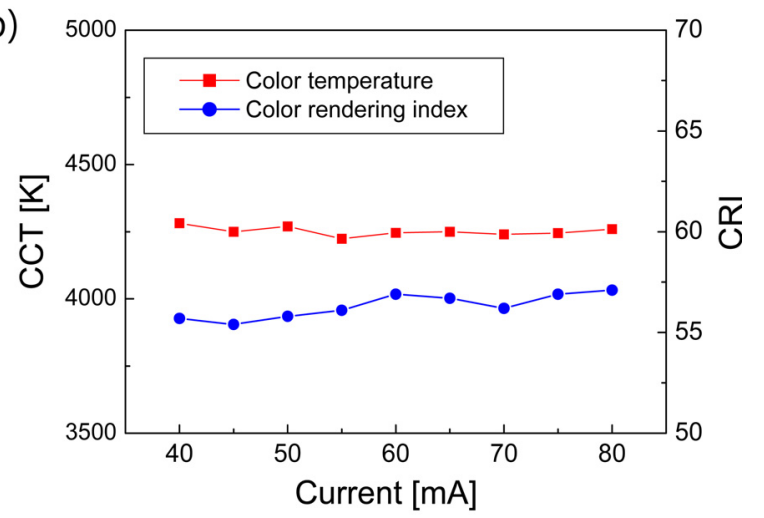

FIG. 4. Color characteristics of the white light source as current increases from 40 to $80 \mathrm{~mA}$. (a) Chromaticity coordinates in the CIE $x y$ chromaticity diagram, (b) correlated color temperature (CCT) and color rendering index (CRI). (a)

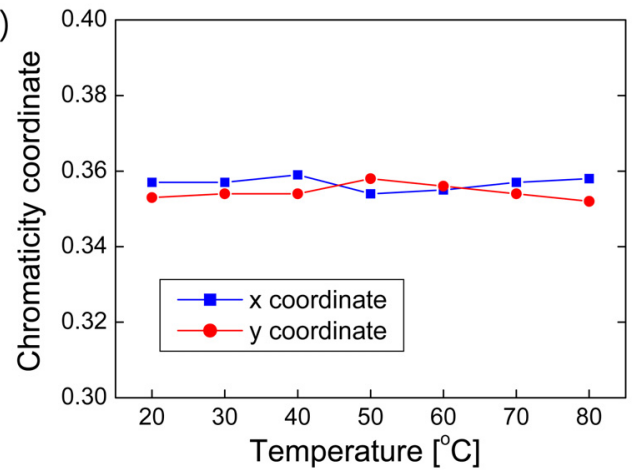

(b)

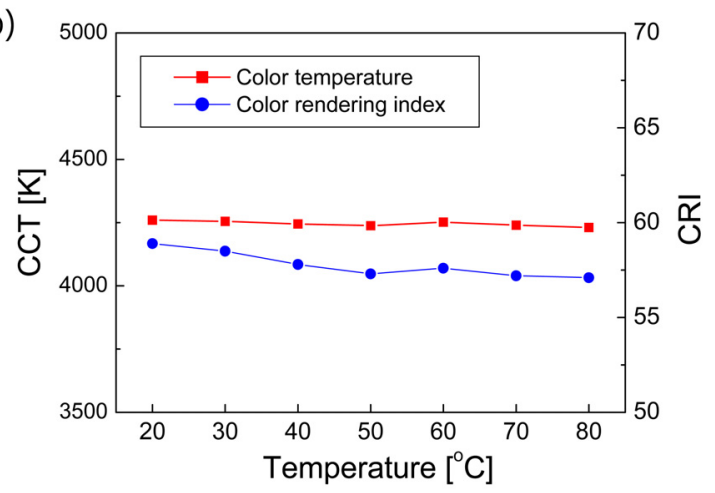

FIG. 5. Color characteristics of the white light source as temperature increases from 20 to $80^{\circ} \mathrm{C}$. (a) Chromaticity coordinates in the CIE $x y$ chromaticity diagram, (b) CCT and CRI.

region corresponding to the sharp laser light spectrum.

As one can see from Fig. 4 and 5, the chromaticity coordinates, CCT, and CRI are stable to the change of current and temperature of the LD. The ratio of the standard deviation to the average value of color parameters is $0.2 \sim$ $1.2 \%$ as current and temperature varies. Fluctuations of the color parameters with current or temperature are attributed to the variation of phosphor conversion efficiency, the instability of LD output, or environmental noise. The stable color characteristics imply that the spectral shape of the white light does not change much with current and temperature, which is due to the spatial separation of the white light emission region and the LD where most of heat is generated. In usual white LEDs, especially for multichip LEDs, color characteristics can be changed significantly with temperature [1], which may cause problems in some illumination applications. On the contrary, the LD-based white light does not have such a temperaturerelated problem. Therefore, it is expected that the demonstrated white light source will find some important applications where stable color characteristics are required.

\section{CONCLUSION}

We demonstrated a phosphor-conversion white light source based on an InGaN-based blue LD emitting at 445 
nm and a yellow phosphor. By combining the blue laser light and yellow light from the phosphor excited by the blue LD, high-brightness white light was realized. When the injection current of the LD was $100 \mathrm{~mA}$, the luminous flux and the luminous efficiency of the white light source were found to be over $5 \mathrm{~lm}$ and $10 \mathrm{~lm} / \mathrm{W}$, respectively. The luminous efficiency of the LD-based white light source increased sublinearly with current. The luminance of the white light source was estimated to be $>10 \mathrm{Mcd} / \mathrm{cm}^{2}$, suitable for the high-brightness illumination application. In addition, color characteristics such as chromaticity coordinates, correlate color temperature, and color rendering index were found to be quite stable to the change of injection current or temperature of the LD.

\section{ACKNOWLEDGMENT}

This work was supported by National Research Foundation of Korea Grant funded by the Korean Government (20100015297).

\section{REFERENCES}

1. E. F. Schubert and J. K. Kim, "Solid-state light sources getting smart," Science 308, 1274-1278 (2005).

2. M. R. Krames, O. B. Shchekin, R. Mueller-Mach, G. O. Mueller, L. Zhou, G. O. Mueller, L. Zhou, G. Harbers, and M. G. Craford, "Status and future of high-power lightemitting diodes for solid-state lighting," IEEE J. Display Technol. 3, 160-175 (2007).

3. G. Chen, M. Craven, A. Kim, A. Munkholm, S. Watanabe, M. Camras, W. Gotz, and F. Steranka, "Performance of high-power III-nitride light emitting diodes," Phys. Status Solidi A 205, 1086-1092 (2008).

4. A. Laubsch, M. Sabathil, J. Baur, M. Peter, and B. Hahn, "High-power and high-efficiency InGaN-based light emitters," IEEE Trans. Electron Devices 57, 79-87 (2010).

5. D. S. Ho, E. H. Kim, I. D. Hwang, K. Shin, J. T. Oh, and B. M. Kim, "Optical skin-fat measurement using miniaturized chip LEDs: a preliminary human study," J. Opt. Soc. Korea 13, 304-309 (2009).

6. Y. Narukawa, I. Niki, K. Izuno, M. Yamada, Y. Murazaki, and T. Mukai, "Phosphor-conversion white light emitting diode using InGaN near-ultraviolet chip,” Jpn. J. Appl. Phys. 41, L371-L373 (2002).

7. Y. Xu, L. Chen, Y. Li, G. Song, and Y. Wang, "Phosphorconversion white light using InGaN ultraviolet laser diode," Appl. Phys. Lett. 92, 021129 (2008).

8. T. Kozaki, S. Nagahama, and T. Mukai, "Recent progress of high-power GaN-based laser diodes," Proc. SPIE 6485, 648503 (2007).

9. H. Y. Ryu, K. H. Ha, S. N. Lee, T. Jang, J. K. Son, H. S. Paek, Y. J. Sung, H. K. Kim, K. S. Kim, O. H. Nam, Y. Park, and J. I. Shim, "High-performance blue InGaN laser diodes with single-quantum-well active layers," IEEE Photon. Technol. Lett. 19, 1717-1719 (2007).

10. H. Y. Ryu, K. H. Ha, J. K. Son, S. N. Lee, H. S. Paek, T. Jang, Y. J. Sung, K. S. Kim, H. K. Kim, Y. Park, and O. H. Nam, "Determination of internal parameters in blue InGaN laser diodes by the measurement of cavity-length dependent characteristics," Appl. Phys. Lett. 93, 011105 (2008).

11. D. W. Lim, H. U. Cho, H. K. Sung, J. C. Yi, and Y. M. Jhon, "A PSPICE circuit modeling of strained AlGaInN laser diode based on the multilevel rate equations," J. Opt. Soc. Korea 13, 386-391 (2009).

12. Ocean Optics, Inc., http://www.oceanoptics.com/products/ spectroradiometric.asp

13. M. H. Kim, M. F. Schubert, Q. Dai, J. K. Kim, E. F. Schubert, J. Piprek, and Y. Park, "Origin of efficiency droop in GaN-based light-emitting diodes," Appl. Phys. Lett. 91, 183507 (2007).

14. Y. C. Shen, G. O. Mueller, S. Watanabe, N. F. Gardner, A. Munkholm, and M. R. Krames, "Auger recombination in InGaN measured by photoluminescence," Appl. Phys. Lett. 91, 141101 (2007).

15. H. Y. Ryu, H. S. Kim, and J. I. Shim, "Rate equation analysis of efficiency droop in InGaN light-emitting diodes," Appl. Phys. Lett. 95, 081114 (2009).

16. J. Hader, J. V. Moloney, and S. W. Koch, "Density-activated defect recombination as a possible explanation for the efficiency droop in GaN-based diodes," Appl. Phys. Lett. 96, 221106 (2010).

17. A. Michiue, T. Miyoshi, T. Yanamoto, T. Kozaki, S. Nagahama, Y. Narukawa, M. Sano, T. Yamada, and T. Mukai, "Recent development of nitride LEDs and LDs," Proc. SPIE 7216, $72161 \mathrm{Z}$ (2009).

18. N. Ohta and A. Robertson, Colorimetry: Fundamentals and Applications (Wiley, Chichester, England, 2006), Chapter 1. 